\title{
Interview with George E. Andrews
}

\section{Toufik Mansour}

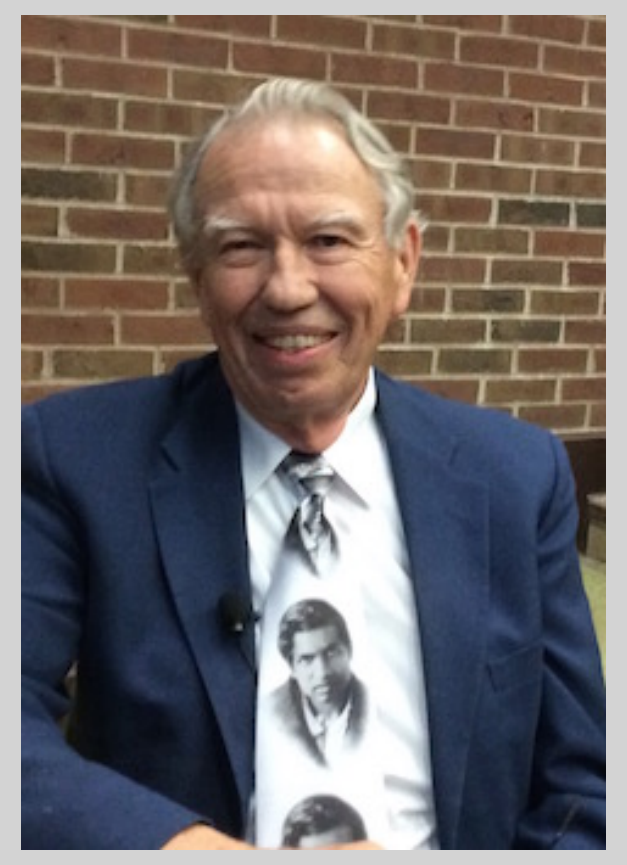

Photo by Vladimir and Mariana Tonchev

George Andrews completed his undergraduate studies at Oregon State University and obtained his Ph.D. at the University of Pennsylvania in 1964 under the direction of Hans Rademacher. He is currently an Evan Pugh Professor of Mathematics at Pennsylvania State University. Professor Andrews held visiting positions at 15 universities. We list some of them: Massachusetts Institute of Technology, University of Wisconsin, University of Waterloo, University of Strasbourg, University of Melbourne, Johannes Kepler University. Professor Andrews has given talks at many conferences, including an invited talk at the International Congress of Mathematicians, in Berlin in 1998. Andrews has received several awards and honors: In 2003 he was elected a member of the National Academy of Sciences. In 1997, he was elected a Fellow of the American Academy of Arts and Sciences. In 2012 he became a fellow of the American Mathematical Society. He was given honorary doctorates from the University of Parma in 1998, the University of Florida in 2002, the University of Waterloo in 2004, SASTRA University in Kumbakonam, India in 2012, and the University of Illinois at Urbana-Champaign in 2014. Professor Andrews is a member of the editorial board for numerous journals, including Advances in Mathematics, Journal of Combinatorial Theory, Series A, Discrete Mathematics, the Electronic Journal of Combinatorics, the Ramanujan Journal. He was one of the Managing Editors of Annals of Combinatorics.

Mansour: Professor Andrews, first of all, we would like to thank you for accepting this interview. Would you tell us broadly what combinatorics is?

Andrews: I view combinatorics as the study of the enumeration of and elucidation of properties of various sets. However, I really think of myself as more of a Combinatory Analyst. This idea is suggested by the title of $\mathrm{P}$. A. MacMahon's magnum opus: "Combinatory Analysis ${ }^{1}$." It is in MacMahon's world where I feel most at home.

Mansour: What do you think about the de- velopment of the relations between combinatorics and the rest of mathematics?

Andrews: I believe there is plenty of user interaction. Often researchers in other areas of mathematics have consulted me, and, as a result, I have been led to new and fruitful combinatorial topics.

Mansour: What have been some of the main goals of your research?

Andrews: I really do not think in terms of "main goals." I regard my research as organic. I am always on the lookout for something that is both new and to which I might be able to

\footnotetext{
The authors: Released under the CC BY-ND license (International 4.0), Published: March 12, 2021

Toufik Mansour is a professor of mathematics at the University of Haifa, Israel. His email address is tmansour@univ.haifa.ac.il

${ }^{1}$ P. A. MacMahon, Combinatory analysis, Cambridge, The University Press, 1915-16.
} 
contribute. I do not have a plan for how things should turn out. Occasionally I have formulated a general program only to see it quickly disappear as the research takes an unexpected turn. I have always tried to follow my rule: Try to hear what the mathematics is trying to tell you!

Mansour: We would like to ask you about your formative years. What were your early experiences with mathematics? Did that happen under the influence of your family or some other people?

Andrews: I was always good at mathematics in elementary school and high school, but it did not interest me. When I was a junior in high school, I had the choice of taking trigonometry or playing in the band. I played in the band. When I was a senior, I went to the guidance counselor for advice on a career choice. He told me that no job is ever really interesting; so, do something that will make money. He noted that I was good in math and science and had an interest in law. So, he suggested that I study to become a patent attorney. I took his advice and enrolled in electrical engineering at Oregon State University with the idea that I would subsequently attend law school. My engineering courses went well as long as they were mathematical. However, in my sophomore year, I was immersed in laboratory work where nothing ever turned out right. In happy contrast, the instructor for many of my courses in my freshman and sophomore years was Harry Goheen, a charismatic proselytizer for mathematics. I entered my junior year as a math major.

Mansour: Were there specific problems that made you first interested in combinatorics?

Andrews: Actually, at the beginning of my career at least through the first few years after graduate school, I viewed myself as a number theorist. It only slowly dawned on me that my work is more combinatorial than number-theoretic. Hans Rademacher's yearlong course on analytic number theory is what lured me into the theory of partitions.

Mansour: What was the reason you chose the University of Pennsylvania for your Ph.D. and your advisor Hans Rademacher?

Andrews: I had decided after my M.A. at Oregon State and my yearlong Fulbright at the University of Cambridge that I wanted to study analytic number theory at the University of Illinois under the guidance of Paul Bateman starting in 1961. As it turned out, Bateman was visiting the University of Pennsylvania during the 1961-62 academic year which was devoted to honoring Hans Rademacher on this 70th birthday. So, I went to Penn because Bateman was there for that year. I assumed that after the year was over, I would transfer to Illinois. Hans Rademacher's course on analytic number theory (mostly about partitions) was magical and completely hooked me. My office mates pointed out that Bateman was Rademacher's former student; so, should not I go to the source for my Ph.D. work? I was persuaded and asked Rademacher if I could be his student. He accepted me. He was a wonderful man in many ways. Although seemingly a formal European academic, he had a mischievous sense of humor. As we walked into my Ph.D. thesis defense, he remarked, "This is our last chance to sink your boat!"

Mansour: What was the problem you worked on in your thesis?

Andrews: Rademacher asked me to improve the work of his former student, Leila Dragonette, on the asymptotics for Ramanujan's third order mock theta functions,

Mansour: What would guide you in your research? A general theoretical question or a specific problem?

Andrews: As I indicated previously, I try to proceed organically. Sometimes this leads to the development of a theory. Sometimes a specific problem obsesses me. Generally, with the latter, I hope that a general theory might follow.

Mansour: When you are working on a problem, do you feel that something is true even before you have the proof?

Andrews: Not only do I feel something is true before I have the proof, but also this is the working hypothesis of every research mathematician (with the possible exception of large cardinal theorists). The only reason to deny this is the fear of appearing unsophisticated at cocktail parties.

Mansour: What three results do you consider the most influential in combinatorics during the last thirty years?

Andrews: Combinatorics is such a diverse subject that I would only be subjectively re- 
calling three striking achievements if I answer directly. No matter which three I would choose there would be a vast portion of combinatorics that would not have been influenced by any of them. I would say that the various algorithms that have been developed for computer algebra packages (Macsyma, Maple Mathematica,...) have had an immense impact across the subject. Also, see my answer to $(*)$.

Mansour: What are the top three open questions in your list?

Andrews: This is much easier than previous question because it refers to "my list." The problems that most interest me (there are many more than 3) are the conjectured RogersRamanujan type partition identities where the relevant generating function is not a modular form. See, for example, the work of S. Kanade and M. C. Russell ${ }^{2}$. Some of their conjectures involve modular forms, and some do not. Bringmann, Mahlburg, Jennings-Shaffer ${ }^{3}$, and Rosengren ${ }^{4}$ have had some success with studying these results, but there is no over-arching theory comparable to what is available when the generating function in question is modular. These conjectures are reminiscent of what is now called the "Big Göllnitz Theorem" ${ }^{5}$." There are at least five proofs of this result now, and yet it is as mysterious as the day Goellnitz discovered it.

Mansour: What kind of mathematics would you like to see in the next ten-to-twenty years as the continuation of your work?

Andrews: The subject will be developed in unpredictable ways that will depend on the brilliance and ingenuity of the people entering the field. This organic development is both healthy and inevitable. I hope to continue to participate for some time to come.

Mansour: Do you think that there are core or mainstream areas in mathematics? Are some topics more important than others?

Andrews: "Core" and "mainstream" are fluid terms that depend heavily both on the achievements within the various branches of mathematics as well as the ways in which these results are applied both within mathematics and in science more generally. At any given moment, some topics are more important than others, and winners and losers will change over time.

Mansour: What do you think about the distinction between pure and applied mathematics that some people focus on? Is it meaningful at all in your case? How do you see the relationship between so-called "pure" and "applied" mathematics?

Andrews: Of course, pure and applied mathematics are distinct. Pure mathematicians study mathematics for its own sake. Applied mathematicians study mathematics with the object of applying it to other branches of science. Each owes much to the other. As my friend and collaborator, the physicist Rodney Baxter remarked: "The problem with splitting a mathematics department up into a pure department and an applied department is that it causes each of them to go off in the wrong direction." The interaction of pure and applied is essential to the vitality of mathematics generally. Encouragement of this is a good thing. Mansour: What advice would you give to young people thinking about pursuing a research career in mathematics?

Andrews: If you are not in love with mathematics, choose another career. There are sufficient uncertainties in the world today that no one can be certain of what career prospects in mathematics will look like in the coming decades. If this is your passion, then mathematics is right for you.

Mansour: Would you tell us about your interests besides mathematics?

Andrews: The biggest interest in my life, by far, is my wife and family. Concerning hobbies, I juggle a little and badly. I play boogiewoogie piano ${ }^{6}$. I greatly enjoy long walks with my wife, and she has interested me in gardening. Philately and golf are past hobbies.

Mansour: You have had a long successful academic career. We see from your nonmathematical writings that you care a lot about mathematics education. What are major issues in the university education system today, specifically

\footnotetext{
${ }^{2}$ S. Kanade and M. C. Russell, IdentityFinder and some new identities of Rogers-Ramanujan type, Exp. Math. 24 (2015), $419-423$.

${ }^{3}$ K. Bringmann, C, Jennings-Shaffer, and K. Mahlburg, Proofs and reductions of various conjectures partition identities of Kanade and Russell, J. reine and ungew. Math. 766 (2020), 109-135.

${ }^{4}$ H. Rosengren, Proofs of some partition identities conjectured by Kanade and Russell, arXiv:1912.03689.

${ }^{5}$ H. Göllnitz, Partitionen mit Differenzenbedingungen, J. Reine Angew. Math. 225 (1967), 154-190.

${ }^{6}$ See https://www .youtube.com/embed/GfXhgGURCxQ.
} 
in mathematics? Do you have any suggestions for rebuilding it?

Andrews: I think I have opposed almost all mathematics educational reform movements starting with the New Math in the 1960s. At the height of the calculus reform movement, I gave talks with the following titles: "Mathematics Educational Reform - Can It Be Stopped Before It's Too Late," and "Calculus Reform: Disintegration By Parts." (The latter title was supplied by a friend who wishes to remain anonymous.) Mathematics is the most rigorous of all the sciences, but teaching mathematics is an art and not a science. Consequently, what we need in the classroom are people who love both mathematics and students. They should learn to teach through lengthy apprenticeships. Currently, at least in the United States, there are truly misguided efforts at all levels of education to label mathematics as "racist" and "white supremacist." The situation has been cogently described by Sergiu Klainerman ${ }^{7}$. This is a much more dire situation than any of the past "Math Wars."

Mansour: Before we close this interview with one of the foremost experts in combinatorics, we would like to ask some more specific mathematical questions. You discovered Ramanujan's lost notebook in 1976 and Bruce Berndt later commented on it as "The discovery of this 'Lost Notebook' caused roughly as much stir in the mathematical world as the discovery of Beethoven's tenth symphony would cause in the musical world". Would you tell us about this discovery and the mathematical importance of this manuscript? Overall, what do you think about the mathematics of Ramanujan?

Andrews: In 1976, I was on leave from Penn State spending the year at the University of Wisconsin as the guest of my late good friend, Richard Askey. In the spring, I was invited to a combinatorics conference in Strasbourg. At the time, the fares for flights to Europe were computed as follows: if you stayed in Europe for at least 3 weeks the fare was $\epsilon$, and if you stayed less than 3 weeks it was $1 / \epsilon$. So, I needed a valid academic excuse to stay in Europe for 3 weeks to attend a week-long conference. I had been informed by the late Lucy Slater that there were a number of G. N. Wat- son's papers that had been contributed to the Trinity College Library in Cambridge. I was quite familiar with Watson's work on explaining some of Ramanujan's discoveries. So, I proposed that, after the Strasbourg conference, I would go to Cambridge to study Watson's notes. When I opened the box containing these papers. I found a number of things related to Ramanujan. In particular, there was a 100 plus pages manuscript labeled "a manuscript of Ramanujan." There are very few words on these pages, mostly formulas and computations. Notably, the phrase "mock theta function" does not appear. However, if you had the uniquely good fortune to write your Ph.D. thesis on mock theta functions, you would discover quickly that the mock theta functions appeared extensively in this manuscript. Now recall that four months before he died in 1920, Ramanujan wrote to Hardy saying, "I have discovered some very interesting functions recently which I call mock theta functions..." So, it became clear, in a matter of minutes, that this manuscript, which had been ignored for 50 years, actually contained the last work of Ramanujan when he was slowly dying but at the height of his intellectual powers. These formulas, all stated without proof, have led in recent years to an explosion of work on mock theta functions connecting them to harmonic weak Maass forms. Bruce Berndt and I have spent more than two decades producing an edited version of Ramanujan's Lost Notebook in five volumes.

Ramanujan was a "magical genius" to use the designation coined by Mark Kac. In Kac's words: "In science, as well as in other fields of human endeavor, there are two kinds of geniuses: the 'ordinary' and the 'magicians.' An ordinary genius is a fellow that you and I would be just as good as if we were only many times better. There is no mystery as to how his mind works. Once we understand what he has done, we feel certain that we, too, could have done it. It is different from the magicians. They are, to use mathematical jargon, in the orthogonal complement of where we are and the working of their minds is for all intents and purposes incomprehensible. Even after we understand what they have done, the process by which they have done it is completely dark."

\footnotetext{
${ }^{7}$ See https://bariweiss. substack. com/p/there-is-no-such-thing-as-white-math.
} 
Mansour: The study of 'integer partitions' is in the core of your work. Why is it interesting to study them? Would you mention some of their applications and connections to other research fields?

Andrews: Partitions have fascinated me since I happened on the footnote on page 2025 of the fourth volume of The World Of Mathematics by James R. Newman (a gift to me from my future wife when I was a freshman in college). Surely integer partitions are the simplest of all topics in number theory; all you need is addition. Here staring me in the face was the incredible Hardy-Ramanujan formula for $p(n)$, the number of partitions of $n$. This first meeting with partitions became a passion with me taking Hans Rademacher's course in 1961-62.

Given the simplicity and ubiquity of addition, it is not surprising to find partitions applied in statistics, statistical mechanics, and computer science.

Mansour: It is easy to understand the idea of integer partitions as it is easy to understand the claim of Fermat's last theorem. We hear the stories about too many amateurs sending their proofs to professional mathematicians, unfortunately always with many flaws, and expecting to become famous by solving such longstanding open problems. But on the search of a resolution for the Fermat's last theorem, many such attempts were also made by good mathematicians. Have you ever received a claimed proof that gives a formula for the number of partitions of a given integer $n$ from some wellknown professional mathematicians?

Andrews: No prominent mathematician has sent me a faulty formula for $p(n)$. I think this is partly because the formula for $p(n)$ is so fantastic that no one is likely to come up with something simple that comes close to the right answer.

Mansour: Bruinier and $\mathrm{Ono}^{8}$ published a paper in Advances in mathematics in 2013, Algebraic formulas for the coefficients of halfintegral weight harmonic weak Maass forms. They derived a formula for the partition function $p(n)$ as a finite sum of algebraic numbers which lie in the usual discriminant $-24 n+1$ ring class field. It has also received a lot of publicity. What can you say about this re- sult? Are there better-closed formulas for integer partition function?

Andrews: The Ono-Bruinier result is a striking and wonderful achievement. When one asks for "better-closed formulas," one must be clear about the meaning of "better." If better means, "mind-blowingly surprising," then Ono and Brunier are definitely the winners. If better means "more suitable for computation," then the Hardy-Ramanujan-Rademacher formula is the place to go. In fact, if one is looking for "charm" in the proof of a formula for $p(n)$, I would suggest Rademacher's second proof using Ford circles.

Mansour: Mock theta functions appear in some of your very recent papers. They also have some interesting applications in physics such as they have been found to be valuable for calculating the entropy of black holes. Why are they important? Would you tell us about some of their interesting applications and point out some possible future research directions?

Andrews: I have said a lot about mock theta functions in answer to previous questions. So, I will only add a few comments here. I have spent a lot of time studying mock theta functions using the approach Ramanujan used. I document this in "How Ramanujan May have Discovered the Mock Theta Functions ${ }^{9}$." The relation of the mock theta functions to harmonic weak Maass forms is now being explored extensively by Ono and others. I do believe that the $q$-series aspect merits more study; often the $q$-series aspect reveals combinatorial truths that are valuable in the application.

Mansour: When we look at the titles of your many papers, we see the terms $q$ identities, $q$-series, $q$-difference equations, $q$ hypergeometric series, $q$-trinomial coefficients, $q$-Catalan numbers, $q$-orthogonal polynomials, and so on. Let us shortly call it $q$-mathematics. Can you tell us about this line of research? What motivates researchers to obtain a $q$ analogue of a 'regular' math concepts?

Andrews: There is this world of " $q$-functions" that is eerily parallel to the classical world of special functions which consists mostly of hypergeometric series. This latter world has been extremely important. In the words of W. W. Sawyer: "In fact, there must be many universi-

\footnotetext{
${ }^{8} \mathrm{~J} . \mathrm{H}$. Bruinier and K. Ono, Algebraic formulas for the coefficients of half-integral weight harmonic weak Maass forms, Adv. in Math. 246 (2013), 198-219.

${ }^{9}$ G. E. Andrews, How Ramanujan may have discovered the mock theta functions, Phil. Trans. R. Soc. A 378 (2020), 20180436.
} 
ties today where 95 percent, if not 100 percent, of the functions considered, are covered by the single symbol $F(a, b ; c ; x)$ [the classical Hypergeometric function]" The $q$-function world is not nearly as well understood and is filled with surprises. For example, two significant results from the classical analysis are: (1) the binomial series, and (2) the beta function. On the surface, they seem unrelated in any significant way. Indeed, the first is an infinite series; the second is integral. In the world of $q$, they are the same thing. In reverse, $q$-theorems sometimes lead to important application in ordinary hypergeometric series theory ${ }^{10}$.

Finally, and most importantly, the $q$-world is the natural home of the generating functions for integer partitions. For example, the celebrated Rogers-Ramanujan identities were first proved in 1894 by Rogers ${ }^{11}$. It took until 1981 when Garsia and Milne published a purely bijective proof in a 50-page paper.

Mansour: (*) At the well-known mathematics forum, Mathoverflow, a post initiated a debate about important formulas in combinatorics. Which ones would you choose as the three most important formulas in combinatorics if you are asked to choose?

Andrews: This is a very hard question for several reasons. First, there are so many meanings of the word "important." Is a result important because it is hard? Does that mean that Fermat's Last Theorem is the most important theorem in mathematics? Consider two papers of Henry Mann: (1) he solved the Artin Conjecture in additive number theory and won the Cole Prize, (2) using elementary methods in the world of $q$, he found the moments for the Wilcoxon signed-rank test in statistics. Which achievement is more important? The first had accumulated 28 citations 60 years later; the second had 2067. I believe that word "important" usually points to some linear order where I only see a rather jumbled partial order.
In addition, I really do not think of myself as a pure combinatorialist. As I alluded to earlier, I think of myself more in the MacMahon mold as a combinatory analyst (perhaps with number theory overtones). I am regularly invited to (1) special function conferences, (2) combinatorics conferences, and (3) number theory conferences. In each, I feel somewhat at home and somewhat of an outsider.

So, I will answer a rephrased question: Name 3 formulas which have been important in my hybrid world that mixes $q$ with enumeration?

(1) Bailey's Lemma: W. N. Bailey in Section 4 of Identities of the Rogers-Ramanujan type $^{12}$ wrote down the complete recipe for what is now called the Strong Form of Bailey's Lemma. However, he was embarrassed by its complexity; so, he only stated "...the most general formulas of basic [i.e. $q$ ] series (apart from those already given) are too involved to be of any general interest." As a result, he missed the most important aspect of his discovery, the Bailey Chain ${ }^{13}$. The Bailey Chain has had a significant impact in the world of $q$ especially including the mock theta functions.

(2) Schur's 1926 partition theorem ${ }^{14}$ : In 1926, I. Schur proved the following "next step" in the study of Rogers-Ramanujan type partition theorems.

Theorem: The number of partitions of $n$ into parts congruent to 1 or 5 modulo 6 equals the number of partitions of $n$ in which the difference between parts is at least 3 and larger than 3 between multiple of 3 .

This theorem and the polynomial generating functions required in several proofs have cropped up again and again in my life. They are related to some orthogonal polynomials in my work with Richard Askey. The AlladiGordon $^{15,16}$ method of weighted words has its genesis in Schur's theorem. Indeed, Schur's theorem is the background behind my collab-

\footnotetext{
${ }^{10}$ C. Krattenthaler and T. Rivoal, An identity of Andrews, multiple integrals, and very-well-poised hypergeometric series, Ramanujan J. 13 (2007), 203-219.

${ }^{11}$ L. J. Rogers, Third Memoir on the Expansion of certain Infinite Products, Proc. London Math. Soc. 26(1) (1994), 15-32

${ }^{12}$ W. N. Bailey, Identities of the Rogers-Ramanujan type, Proc. London Math. Soc. (2) 50 (1948), 1-10.

${ }^{13}$ G. E. Andrews, Multiple series Rogers-Ramanujan type identities, Pac. J. Math. 114 (1984), $267-283$.

${ }^{14}$ I. Schur, Zur additiven Zahlentheorie, S.-B. Preuss. Akad. Wiss. Phys.-Math. KL, 1926, pp. 488-495. (Reprinted in I. Schur, Gesammelte Abhandlungen, vol. 3, Springer, Berlin, 1973, pp. 43-50.

${ }^{15}$ K. Alladi and B. Gordon, Generalizations of Schur's partition theorem, Manuscripta Math. 79 (1993), $113-126$.

${ }^{16} \mathrm{~K}$. Alladi and B. Gordon, Schur's partition theorem, companions, refinements and generalizations, Trans. Amer. Math. Soc. 347 (1995), 1591-1608.

${ }^{17}$ K. Alladi, G. Andrews, and A. Berkovich, A new four parameter q-series identity and its partition implications, Invent. Math. 153 (2003), 231-260.
} 
oration with Alladi and Berkovich ${ }^{17}$. Schur's theorem also fits nicely into the Bailey Chain machinery, and one proof involves the $q$ trinomial coefficients that arose in my physics collaboration with Rodney Baxter ${ }^{18}$.

(3) Jacobi's Triple Product: I chose this because it is ubiquitous in the $q$ world. It is the quintessence of elegance, and my first $q$ paper was: A simple proof of Jacobi's triple product identity $^{19}$.

Mansour: In your research, you have extensively used combinatorial reasoning to address many important problems. How do enumerative techniques engage in your work?

Andrews: Most of my work is a mix of analysis and enumeration. So my standard approach is to use elementary, combinatorial arguments to get the matter under consideration into analytic terms where the real heavy weather sets in. I have written a few papers that are almost completely enumerative, but this is the exception rather than the rule.

Mansour: Would you tell us about your thought process for the proof of one of your favorite results? How did you become interested in that problem? How long did it take you to figure out a proof? Did you have a "eureka moment"?

Andrews: There are many favorite results. So, I will choose the discovery of what is now called the Andrews-Gordon theorem. In 1961, Basil Gordon found a stunning generalization of the Rogers-Ramanjan identities. It was stated in purely partition-theoretic terms. Now the Rogers-Ramanujan identities were originally stated as an analytic series-product identity, and it was obviously appealing to find the analytic series-product version of Gordon's theorem. The Rogers-Ramanujan identities are linked to the modulus 5, and there were further known results of this nature linked to 7 and 9 , but nothing seemed to generalize to $2 k+1$. I tried a number of approaches in the late 1960s and early '70s. I actually found the initial stages of the right answer several years before I published it. I had expected to get something looking like the cases at 7 and 9, and my new results looked nothing like them; so, I set this aside. Thus the "aha" moment arrived a couple of years later when I realized that my previous work actually would lead to the Rogers-Ramanujan generalization that was "in the book."

Mansour: Is there a specific problem you have been working on for many years? What progress have you made?

Andrews: I have, for the last 3 years worked on and off on conjecture I4 in the paper by S. Kanade and M. C. Russell ${ }^{2}$. It is a RogersRamanujan type identity related to the modulus 9. I made great progress in the first halfhour that I worked on it, but progress has been rather minimal since then. My efforts on this problem have led me to a number of other theorems that please me greatly, but I am no closer to proving this conjecture than I was in 2018. I would note that the best thing about this effort is all of the resulting, unexpected, tangential discoveries.

Mansour: Do you keep a secret notebook that will lead to many flourishing research programs when it will have been discovered in the future?

Andrews: I have no secret notebooks. However, I am something of a packrat. So, I have many file cabinets devoted to the background work I have done on my $340+$ papers. Many of them are so idiosyncratic that I am quite sure no one will get much out of them. Often, I myself need to look up something in them and have a hard time deciphering my old scribblings.

Mansour: Professor George E. Andrews, I would like to thank you for this very interesting interview on behalf of the journal Enumerative Combinatorics and Applications.

\footnotetext{
${ }^{18}$ G. E. Andrews and R. J. Baxter, Lattice gas generalization of the hard hexagon model. III. q-Trinomial coefficients, J. Stat. Phys. 47 (1987), 297-330.

${ }^{19}$ G. E. Andrews, A simple proof of Jacobi's triple product identity, Proc. Amer. Math. Soc. 16 (1965), 333-334.
} 